Article

\title{
FTn Finite Volume Analysis of Ultrafast Laser Radiation Transport through Human Skin Cancer
}

\author{
Kamel Guedri ${ }^{1,2, *}$, Shougi Suliman Abosuliman ${ }^{3}$ and Mowffaq Oreijah ${ }^{1}$ \\ 1 Mechanical Engineering Department, College of Engineering and Islamic Architecture, \\ Umm Al-Qura University, B. Po 5555, Makkah 21955, Saudi Arabia; mmoreijah@uqu.edu.sa \\ 2 UR: « Matériaux, Energie et Energies Renouvelables » (MEER), Faculty of Sciences of Gafsa, \\ B.P.19-2112 Zarroug, Gafsa 2100, Tunisia \\ 3 Department of Port and Maritime Transport, Maritime Studies Faculty, King Abdulaziz University, \\ Jeddah 21589, Saudi Arabia; sabusulaiman@kau.edu.sa \\ * Correspondence: kamel.guedri@enim.rnu.tn; Tel.: +966-563-884-798
}

Received: 6 September 2020; Accepted: 9 October 2020; Published: 12 October 2020

Featured Application: A numerical tool is proposed for an early detection of skin cancer based on a 3D numerical transient radiative heat transfer study of the ultrafast-laser transport through normal and malignant human skins.

\begin{abstract}
In this work, we develop a numerical tool for the early detection of skin cancer using a 3D numerical transient radiative heat transfer study of ultrafast-laser transport through normal and malignant human skins. The curved-line advection method (CLAM) spatial scheme and the FTn angular scheme of the finite volume method (FVM) are investigated to analyze the above-cited physical phenomena. Both Fresnel specular and diffuse boundary conditions are analyzed. Human skin is considered based on optical properties available from other sources. The temporal radiative signals of skin with malignancies were compared to those of normal skin. Malignancies in the basal layer and epidermis were simulated. Further, the effects of laser light wavelength and the volume of the cancerous region and its scattering coefficient on these signals were studied. The results show that (1) the effect of the Fresnel boundary in the modeling was pronounced; (2) the peak magnitude for human skin with the cutaneous melanoma (CM) had the maximum value in comparison with those obtained for the two other malignancies; and (3) when cancer fully affected the living epidermis with any of these malignancies, the reflectance was more than its predicted value when affected by the basal only.
\end{abstract}

Keywords: radiation of ultrafast laser; malignant human skin; the FTn finite volume method; early detection of skin cancer; Fresnel boundary

\section{Introduction}

Skin cancer arises from the skin due to the development of abnormal cells in the tissue. These cells invade or spread to other regions of the skin or to other human organs. There are three major types of skin cancers. Cutaneous melanoma (CM) is considered the most dangerous among all forms of human skin cancers. On the other hand, nodular basal cell carcinoma (NBCC) and squamous cell carcinoma (SCC) are grouped together as non-melanoma skin cancers. Melanoma and carcinoma are two different types of cancer. They originate from the melanocytes and epithelial tissue, respectively [1,2].

Skin cancers can be effectively treated if they are detected early. However, they can be fatal if left untreated, thus it is important to develop methods for an early optical detection of skin abnormalities. One of the most applicable methods is to subject human skin to an ultrafast-laser pulse to analyze the 
temporal variation. The obtained modifications of the optical properties and conditions lead an early detection of skin cancer. Thus, ultrafast-laser propagation through scattering and participating media has received significant attention in literature, principally due to its importance to several biomedical diagnoses [1].

Some studies of the bio-heat transfer phenomenon are expensive and can become dangerous to living body under testing, especially in cases where an ultrafast laser is used. Further, during irradiation of the tissue by laser, diverse types of interaction may occur, among which thermal effects have a large presence. To study the effects of radiation on biomedical tissues, it is necessary to use the transient radiative transfer equation (TRTE) [2]. A detailed understanding and analysis of bio-heat transfer is required to design a new method that can ensure accurate solutions.

Recently, several numerical techniques have been used to model laser light transport in participating media. The Monte Carlo method [3-5], integral equation (IE) formulation [6] and discrete ordinates method [7-9] have been used to solve the TRTE. In the past few decades, the finite volume method (FVM) has become as one of the most attractive methods for solving radiation transport [10-19]. It has been formulated, tested and applied in several steady and transient radiative transfer problems. However, the standard FVM form suffers from ray effects and false scattering due to the angular and spatial discretization of the TRTE, respectively [10-19].

High-order spatial differencing schemes such as minimal model (MINMOD), Curved-Line Advection Method (CLAM), Monotone Upstream-centered Scheme for Conservation Laws (MUSCL) and sharp and Monotonic Algorithm for Realistic Transport (SMART) may be able to decrease numerical smearing and increase TRTE solution accuracy $[9,14]$. On the other hand, several schemes have recently been proposed to decrease ray effects, including the combined Monte Carlo and finite volume method (MCFVM) [17] and the angular-multiblock procedure [18]. The new non-uniform angular discretization scheme is called the FTn FVM [10-14,19], and three-dimensional FTn FVM results have shown a decrease in ray effects and an increase in TRTE solution accuracy.

Bhowmik et al. [12] recently analyzed the effects of a short-pulse laser on normal skin and malignant human skin. In that study, human skin was modeled as one-dimensional tissue; however, in the present work, a three-dimensional human skin is considered. Additionally, Bhowmik et al. [12] applied the modified discrete ordinate method, whereas in this study a high-order spatial differencing scheme (the CLAM) and the FTn angular scheme of the finite volume method have been combined to study ultrafast-laser transport through normal and malignant human skins. All these improvements have been introduced to reduce numerical smearing and ray effect errors while increasing the solution accuracy of the numerical model. Such a discovery reported here for non-homogenous cells with a finite width and small inhomogeneities may be employed for the early discovery of small malignant or cancerous tumors in human skin. Thus, this paper analyzes the ultrafast laser interaction with nonhomogeneous three-dimensional biomedical tissue, and it offers an efficient numerical tool for an early detection of skin cancer.

\section{Mathematical Formulation}

\subsection{Transient Radiative Transfer Equation (TRTE)}

Assuming that the biological tissue is modeled by an anisotropically participating 3D rectangular media containing inhomogeneities, the ultrafast laser propagation through tissues can be analyzed by the transient radiative transfer equation (TRTE) provided in [20,21]:

$$
\frac{1}{c_{\text {med }}} \frac{\partial I_{d}(r, \Omega, t)}{\partial t}+\frac{\partial I_{d}(s, \Omega, t)}{\partial s}=-\beta I_{d}(s, \Omega, t)+S_{d}(s, \Omega, t)+S_{c}(s, \Omega, t)
$$


where $\beta=\sigma_{a}+\sigma_{s}$ is the extinction coefficient of the medium. The source terms due to the collimated $S_{c}$ and diffuse $S_{d}$ radiation are given as follows:

$$
\begin{gathered}
S_{c}(r, \Omega, t)=\sigma_{a} I_{b}(s, t)+\frac{\sigma_{s}}{4 \pi} \int_{4 \pi} I_{c}\left(s, \Omega^{l^{\prime}}, t\right) \Phi\left(\Omega^{l^{\prime}} \rightarrow \Omega^{l}\right) d \Omega^{l^{\prime}} \\
S_{d}(r, \Omega, t)=\frac{\sigma_{s}}{4 \pi} \int_{4 \pi} I_{d}\left(s, \Omega^{l^{\prime}}, t\right) \Phi\left(\Omega^{l^{\prime}} \rightarrow \Omega^{l}\right) d \Omega^{l^{\prime}}
\end{gathered}
$$

It is noted that the diffuse component of the intensity, $I_{d}$, received at the boundary of incidence marks in the reflectance, where $I=I_{d}+I_{c}$ is the intensity in any general direction $\Omega ; I_{c}$ and $I_{d}$ are the attenuation component of collimated and diffuse radiation, respectively. More descriptions can be found in $[20,21]$.

With incident angle of the laser source $\theta_{c}$ (Figure 1), the intensity of the ultrafast laser, $I_{c}$, has a Gaussian expression in time and is uniform in space as follows [21]:

$$
\begin{gathered}
I_{c}(x=0, y, z, \theta, t)=I_{c, \max }(\theta, t) \exp \left[-\beta d_{c}\right] \times \exp \left[-4 \ln 2\left(\frac{t-\frac{d_{c}}{c_{\text {med }}}-t_{c}}{t_{p}}\right)^{2}\right] \times \\
\delta\left(\theta-\theta_{c}\right) \quad ; 0<t<2 t_{c}
\end{gathered}
$$

where $I_{c, \max }$ denotes the maximum radiation intensity of the laser pulse, which occurs at $t=t_{p} ; t_{p}$ is the full width at half maximum (FWHM); $d_{c}=\mathrm{x} / \cos \theta_{c}$ is the geometric distance; and $T_{p}\left(=2 t_{c}\right), t_{c}=3 t_{p}$ and $\delta$ represent the time period, cut-off time and Dirac-delta function, respectively.

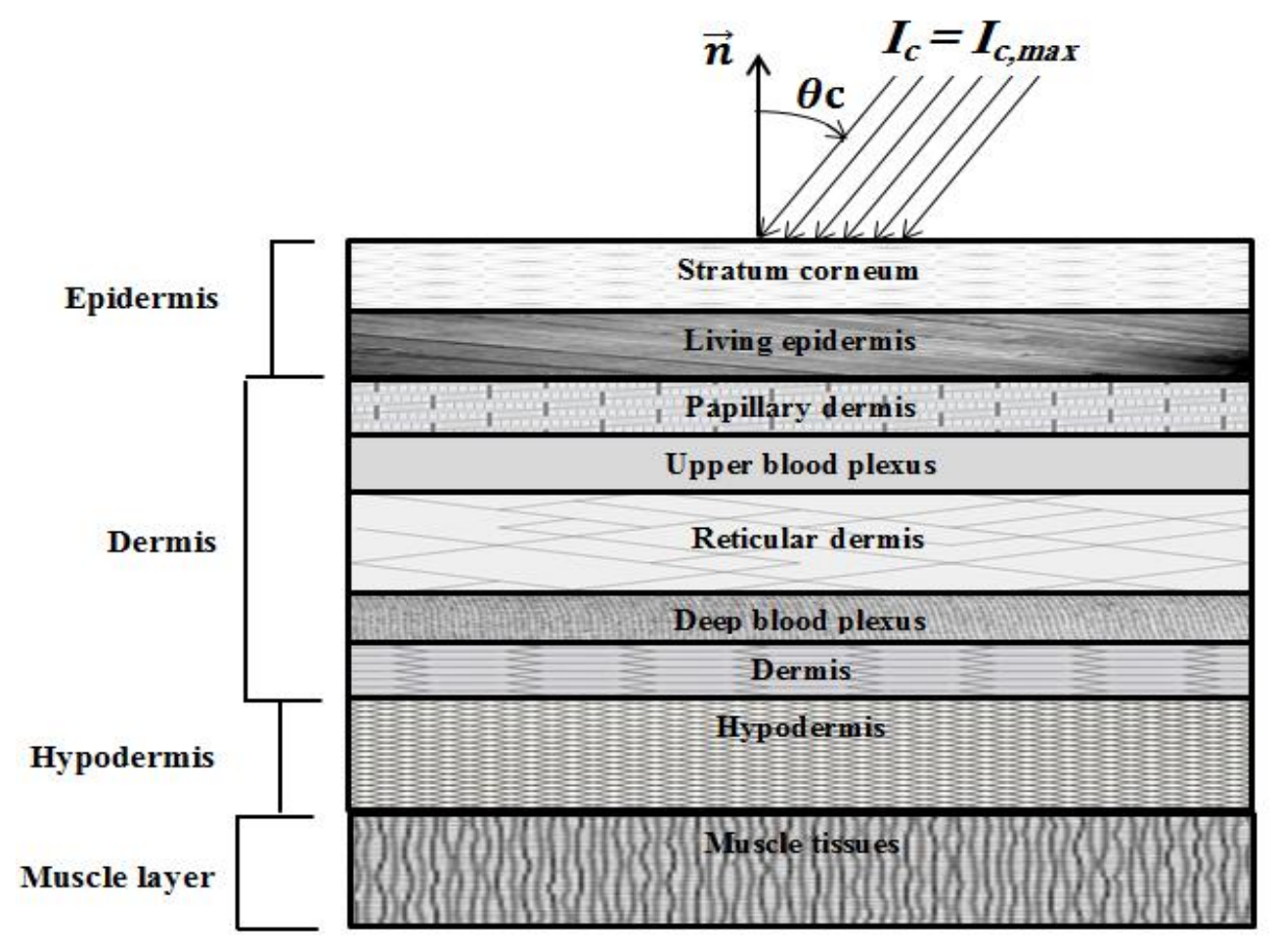

Figure 1. Schematic of the human skin subjected to ultrafast laser.

In the present formulation, both Snell's refraction and Fresnel reflections are considered, as proposed by Guo and Kim [22]. Both diffuse and specular reflection components are treated in the developed model. More details can be found in [22]. 


\subsection{Discretization of Transient Radiative Transfer Equation (TRTE)}

Equation (1) can be expressed (with linear anisotropy) as

$$
\frac{1}{\beta \cdot c_{\text {med }}} \frac{\partial I_{d}^{l}}{\partial t}+\frac{1}{\beta} \frac{\partial I_{d}^{l}}{\partial \mathrm{s}}=-I_{d}^{l}+(1-\omega) I_{b}+\frac{\omega}{4 \pi} \sum_{l^{\prime}=l}^{L} I_{d}^{l} \Phi^{l^{\prime l} l} \Delta \Omega^{l}+S_{c}^{l}
$$

where $L$ defines the total number of discrete solid angles. In the present work, the scattering phase function is expressed as a series in terms of Legendre's polynomial [23]. Thus, the linearized TRTE is given by

$$
\frac{1}{\beta \cdot c_{m d}} \frac{\partial I_{d}^{l}}{\partial t}+\frac{1}{\beta} \frac{\partial I_{d}^{l}}{\partial \mathrm{s}}=-\beta_{m}^{l} I_{d}^{l}+S_{m}^{l}
$$

where the modified extinction coefficient $\beta_{m}$ is given as

$$
\beta_{m}^{l}=\left(1-\frac{\omega}{4 \pi} \Delta \Omega^{l}\right)-\frac{\omega}{4 \pi} g \cos ^{2} \theta^{l} \Delta \Omega^{l}-g \sin ^{2} \theta^{l} \Delta \Omega^{l}
$$

The modified source term is given by

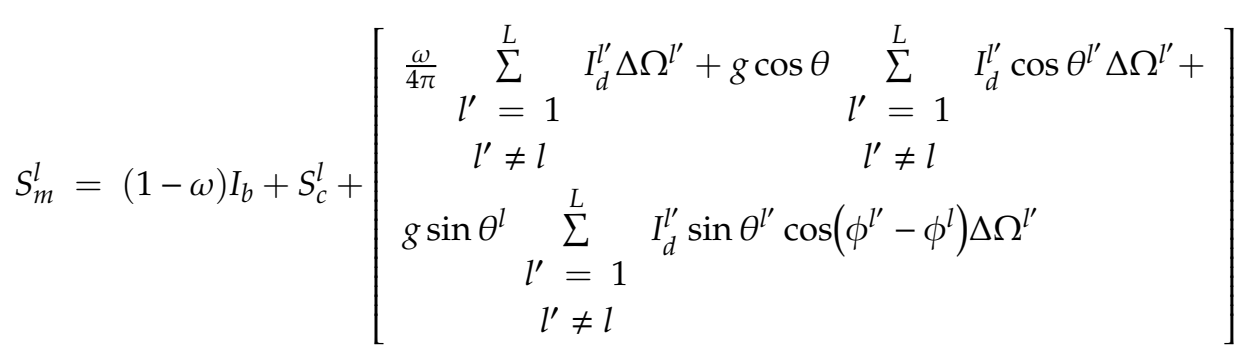

The FTn FVM used $n(n+2)$ control angles where the polar angle was uniformly subdivided into an even number (n). However, a non-uniform subdivision of the azimuthal angle was considered. In fact, for each level of the polar angle, the azimuthal angle was subdivided into the numbers of the following arrangement: 4,8,12 .. 2n,2n ... 12,8,4 [14-16,19].

Different spatial discretization schemes may be applied to relate radiation intensities on the interfaces to the radiation intensity at the grid nodes. The curved-line advection method (CLAM) scheme differs from the STEP scheme by the interpolation of intensities in a specified interface [9-16]. These differences have been analyzed in [9-16].

The forward Euler scheme (or the so-called the explicit scheme) is a first-order numerical procedure in which the temporal dependent variable in the time instant $(t+\Delta t)$ at a given point (e.g., the radiation intensity at time instant $t+\Delta t: I^{t+\Delta t}$ for the TRTE) can be calculated explicitly from the current time instant $(t)$ solution values (e.g., $I^{t}$ for the TRTE) according to (for the TRTE example):

$$
\frac{1}{c_{\text {med }}} \frac{\partial I}{\partial t} \approx \frac{I^{t+\Delta t}-I^{t}}{c_{\text {med }} \Delta t}=F\left(t, I^{t}\right)
$$

where $\Delta t$ is the time step and $F$ is the temporal derivative of $I$ at instant $t$, divided by the propagation speed of light within the medium, $C_{\text {med }}$. This temporal scheme is easier to apply and computationally more efficient than the backward Euler (implicit) scheme, where the solution $I^{t+\Delta t}$ is determined by resolving a set of equations containing both the current and latter state. It is also noted that the explicit scheme needs a smaller time step $\Delta t$, by comparison to the implicit scheme, in order to reach numerical stability. 


\section{Results and Discussions}

Skin cancer represents one of the most common type of cancer in the world. It develops, normally, at the epidermis (the outer layer) of the human skin. Cutaneous melanoma (CM) represents the most serious melanoma form of human skin cancer [21]. Nodular basal cell carcinoma (NBCC) and squamous cell carcinoma (SCC) define the forms of non-melanoma. They occur more frequently and spread at a slower rate than CM melanoma.

Salomatina et al. [24] and Garcia-Uribe et al. [25] proposed different optical properties of normal and cancerous human skin. In vivo characterizations have been employed in their works, and analyses of pigmented skin lesions have been considered using oblique incidence diffuse reflectance spectrometry [25]. In our present work, the main optical properties of non-melanoma and melanoma type skin carcinomas are taken from [24,25]. Firstly, malignancy in human skin start as a thin layer of carcinoma situated in the basal layer of the living epidermis, as explained in [21]. Then, the malignancy spreads into the living epidermis. In the third phase, it enters the stratum corneum, and appears as a tumor at the skin's outer-surface.

Anderson and Parrish [26] presented an optical characterization of normal and malignant human skins in the range of $600-1300 \mathrm{~nm}$ wavelengths. This range of wavelength has a special potential for the optical discovery of skin abnormalities. The thickness, absorption coefficient, scattering coefficient and anisotropic factor of a normal skin model at 520 and $840 \mathrm{~nm}$ are presented in Table 1 [21,27-29], while the same properties corresponding to a wavelength of $785 \mathrm{~nm}$ are summarized in Table $2[21,27,28]$. The optical properties of a skin lesion at $785 \mathrm{~nm}$ are also displayed in Table 3.

Table 1. Thickness, absorption coefficient, scattering coefficient and anisotropic factor of a skin model at 520 and $840 \mathrm{~nm}[21,27-29]$.

\begin{tabular}{|c|c|c|c|c|c|c|c|c|}
\hline \multirow{2}{*}{ Layers } & \multirow{2}{*}{$d(\mathrm{~mm})$} & \multirow{2}{*}{$n$} & \multicolumn{3}{|c|}{$\lambda=520 \mathrm{~nm}$} & \multicolumn{3}{|c|}{$\lambda=840 \mathrm{~nm}$} \\
\hline & & & $\sigma_{a}\left(\mathrm{~mm}^{-1}\right)$ & $\sigma_{s}\left(\mathrm{~mm}^{-1}\right)$ & g & $\sigma_{a}\left(\mathrm{~mm}^{-1}\right)$ & $\sigma_{s}\left(\mathrm{~mm}^{-1}\right)$ & g \\
\hline Stratum corneum & 0.01 & 1.45 & 4.0 & 57.0 & 0.77 & 0.00091 & 17.6125 & 0.8 \\
\hline Living epidermis & 0.08 & 1.4 & 4.0 & 57.0 & 0.77 & 0.13 & 17.6125 & 0.8 \\
\hline Papillary dermis & 0.1 & 1.4 & 0.5 & 50.0 & 0.77 & 0.105 & 10.625 & 0.8 \\
\hline Upper blood plexus & 0.08 & 1.39 & 2.45 & 50.0 & 0.79 & 0.15875 & 15.485 & 0.818 \\
\hline Reticular dermis & 1.50 & 1.4 & 0.5 & 50.0 & 0.77 & 0.105 & 10.625 & 0.8 \\
\hline Deep blood plexus & 0.07 & 1.34 & 18.1 & 50.0 & 0.96 & 0.444 & 46.0625 & 0.962 \\
\hline Dermis & 0.16 & 1.4 & 0.5 & 50.0 & 0.77 & 0.105 & 10.625 & 0.8 \\
\hline Hypodermis & 3.0 & 1.44 & 0.4778 & 33.72 & 0.9 & 0.009 & 11.13 & 0.9 \\
\hline Muscle tissues & 3.0 & 1.37 & 0.1366 & 8.88 & 0.9054 & 0.029 & 6.71 & 0.9 \\
\hline
\end{tabular}

Table 2. Thickness, absorption coefficient, scattering coefficient and anisotropic factor of a skin model at $785 \mathrm{~nm}[21,27-29]$.

\begin{tabular}{cccccc}
\hline Layers & $\boldsymbol{d}(\mathbf{m m})$ & $\boldsymbol{n}$ & $\sigma_{\boldsymbol{a}}\left(\mathbf{m m}^{-1}\right)$ & $\sigma_{\boldsymbol{s}}\left(\mathbf{m m}^{-1}\right)$ & $\mathrm{g}$ \\
\hline Stratum corneum & 0.01 & 1.4 & 0.00089 & 18.95 & 0.8 \\
Living epidermis & 0.08 & 1.4 & 0.19 & 18.95 & 0.8 \\
Papillary dermis & 0.1 & 1.4 & 0.13 & 11.65 & 0.8 \\
Upper blood plexus & 0.08 & 1.39 & 0.15875 & 15.485 & 0.818 \\
Reticular dermis & 1.50 & 1.4 & 0.13 & 11.65 & 0.8 \\
Deep blood plexus & 0.07 & 1.34 & 0.38875 & 46.165 & 0.962 \\
Dermis & 0.16 & 1.4 & 0.13 & 11.65 & 0.8 \\
Hypodermis & 3.0 & 1.44 & 0.008 & 11.44 & 0.9 \\
Muscle tissues & 3.0 & 1.37 & 0.031 & 7.130 & 0.9 \\
Total thickness $(\mathbf{m m})$ & 8.0 & & & & \\
\hline
\end{tabular}


Table 3. Absorption coefficient, scattering coefficient and anisotropic factor of a skin lesion at $785 \mathrm{~nm}[24,25]$.

\begin{tabular}{cccccc}
\hline & Skin Carcinoma & $n$ & $\sigma_{a}\left(\mathbf{m m}^{-1}\right)$ & $\sigma_{s}\left(\mathbf{m m}^{-1}\right)$ & $\mathbf{g}$ \\
\hline \multirow{2}{*}{ Non-melanoma } & Nodular basal cell carcinoma (NBCC) & 1.4 & 0.035 & 8.140 & 0.8 \\
Melanoma & Squamous cell carcinoma (SCC) & 1.4 & 0.062 & 6.680 & 0.8 \\
& Cutaneous melanoma (CM) & 1.4 & 0.0075 & 9.185 & 0.8 \\
\hline
\end{tabular}

In this section, human skin with and without malignancies is numerically studied after having been exposed to a low-power short-pulse (ultrafast) laser (Figures 1 and 2). The incident laser pulse $I_{c}(t)$ obeys Equation (4), and the same thicknesses for both layers (i.e., the epidermis and the dermis) are used as those by Wang et al. [30] and Bhowmik et al. [21]. The thicknesses of the hypodermis (the subcutaneous) and muscular layers and their refractive indices, $n$, are obtained from the works of Bhowmik et al. [21] and Bashkatov et al. $[27,28]$. Thus, the total thickness in the collimated $x$-direction of the human skin layers is $8.0 \mathrm{~mm}$. In our study, three-dimensional skin layers, with dimensions $8 \times 100 \times 100 \mathrm{~mm}^{3}$, are simulated. The total optical thickness $\tau$ at a wavelength of $785 \mathrm{~nm}$ (Table 2) is obtained from its integration along the total thickness of the skin as follows [21]:

$$
\tau=\int_{x=0}^{x=8.0 \mathrm{~mm}}\left(\sigma_{a}+\sigma_{s}\right) d x=83.0
$$

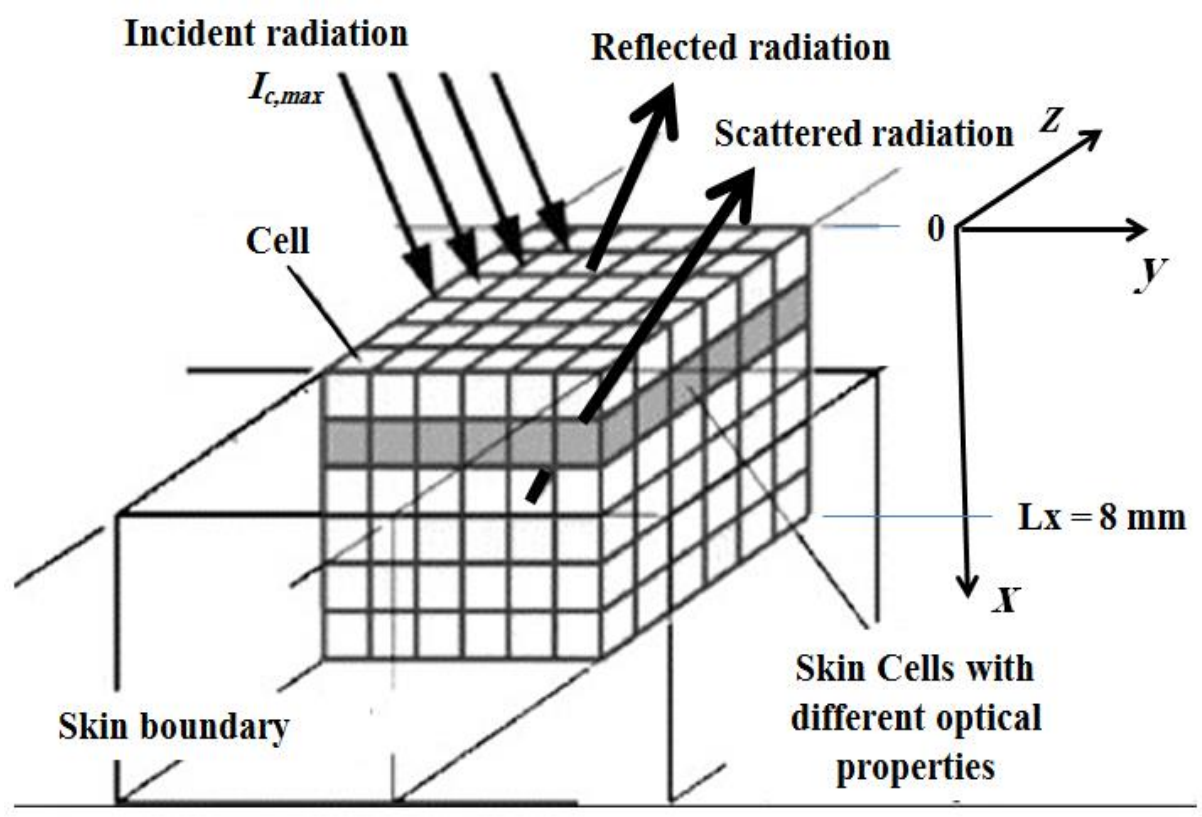

Figure 2. Schematic of the ultrafast laser's interaction within the skin used in the simulation.

The incident laser pulse $I_{c}(t)$ has a Gaussian's profile, as plotted in Figure 3. Temporal deviations of transmittance and reflectance signals are examined for three-dimensional skins. All computations are done using $400 \times 21 \times 21$ control volumes and 80 control angles (i.e., the FT8). Only the predictions of the CLAM scheme are analyzed. For all cases, a time step size $\Delta t=\frac{\Delta x}{n \times c} \approx 9.5 \cdot 10^{-3}$ ps has been applied, and thus this step size is much less than the pulse width (i.e., $(\Delta t \ll t \mathrm{t}))$. The incident angle of the ultrafast laser is taken as normal to the irradiated boundary (i.e., $\theta_{c}=0.0^{\circ}$ ) (Figure 3). For all results, both transmittance and reflectance signals have been normalized according to their corresponding highest values in each corresponding case. 


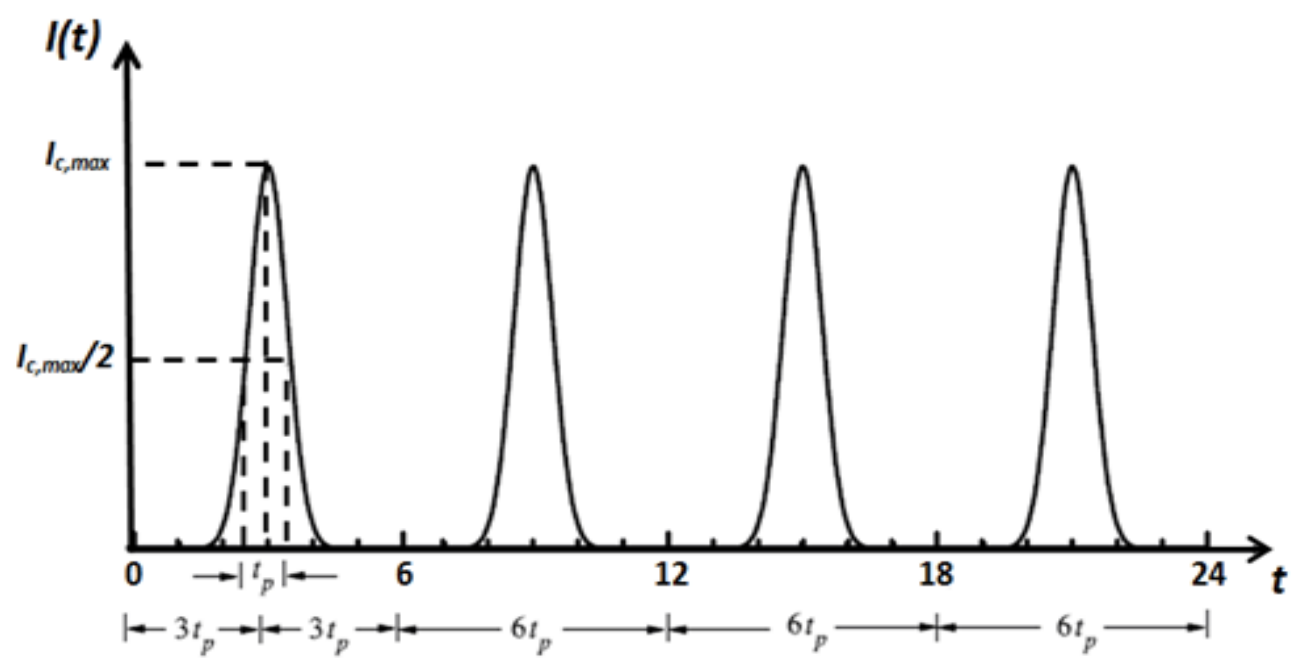

Figure 3. Temporal profile of the incident short-pulse (ultrafast) laser.

\subsection{Human Skin without Malignancies}

In this section, the influence of the pulse width $t_{p}$ on the normalized reflectance and the transmittance for normal human skin is analyzed according to the properties described below as well as without malignancies (Figures 4 and 5). Four pulse widths are considered for a single pulse: $t_{p}=1 \mathrm{~ns}, 100 \mathrm{ps}, 10 \mathrm{ps}$ and 1 ps. In addition, signals reported by Bhowmik et al. [21] are plotted for a one-dimensional case so that we may compare our present three-dimensional results using the FTn FVM to those given by the discrete ordinates method utilized in [21]. In order to analyze the signals properly, we depict the results using the CLAM scheme only. In fact, for fine spatial grids, the results obtained using the STEP scheme, which are not shown here, closely match those reported using the CLAM scheme.
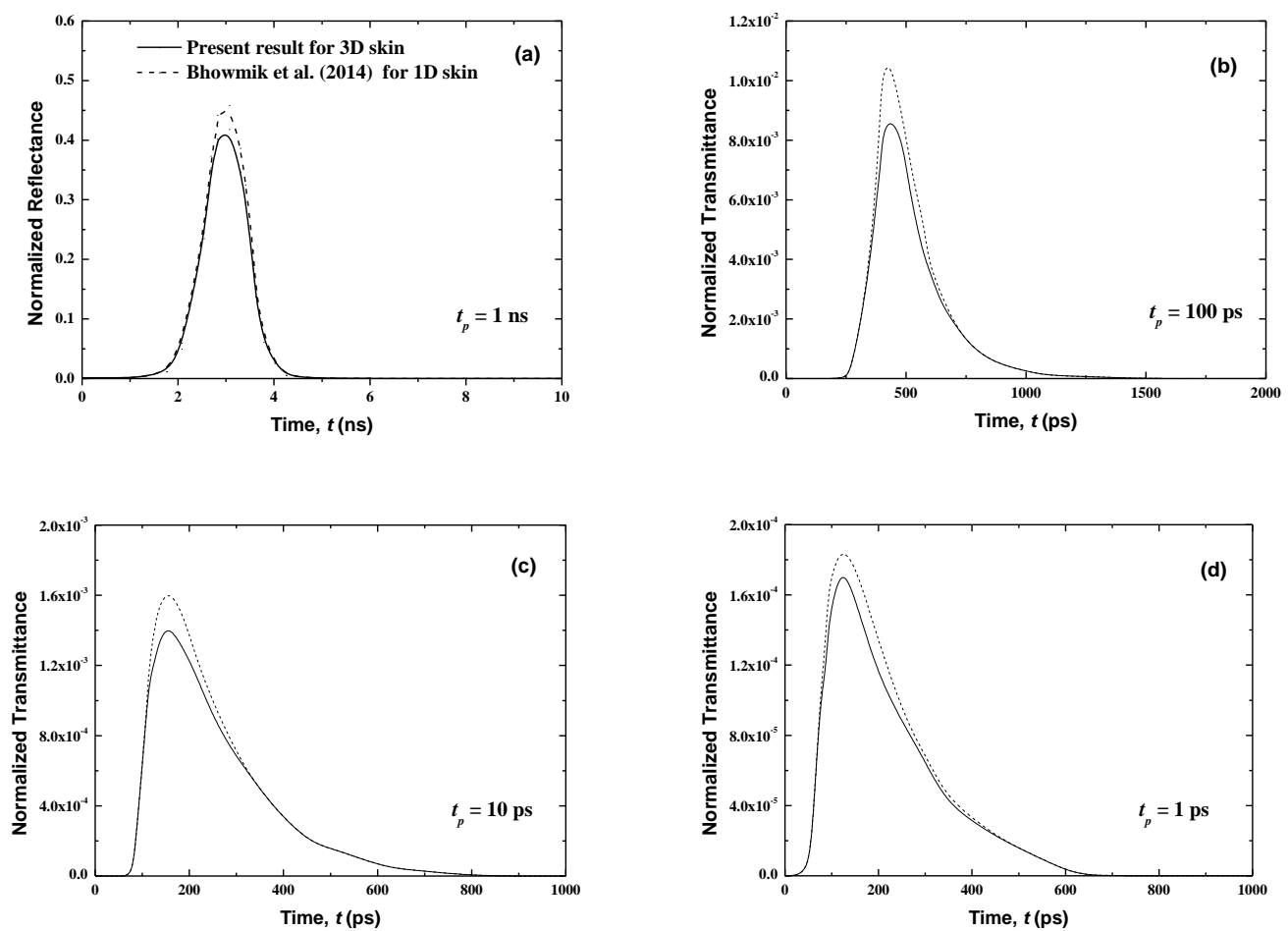

Figure 4. Temporal signals of normalized transmittance from the normal skin subjected to the laser pulse at different pulse widths of (a) $t_{p}=1 \mathrm{~ns},(\mathbf{b}) t_{p}=100 \mathrm{ps},(\mathbf{c}) t_{p}=10 \mathrm{ps}$ and, (d) $t_{p}=1 \mathrm{ps}$. 

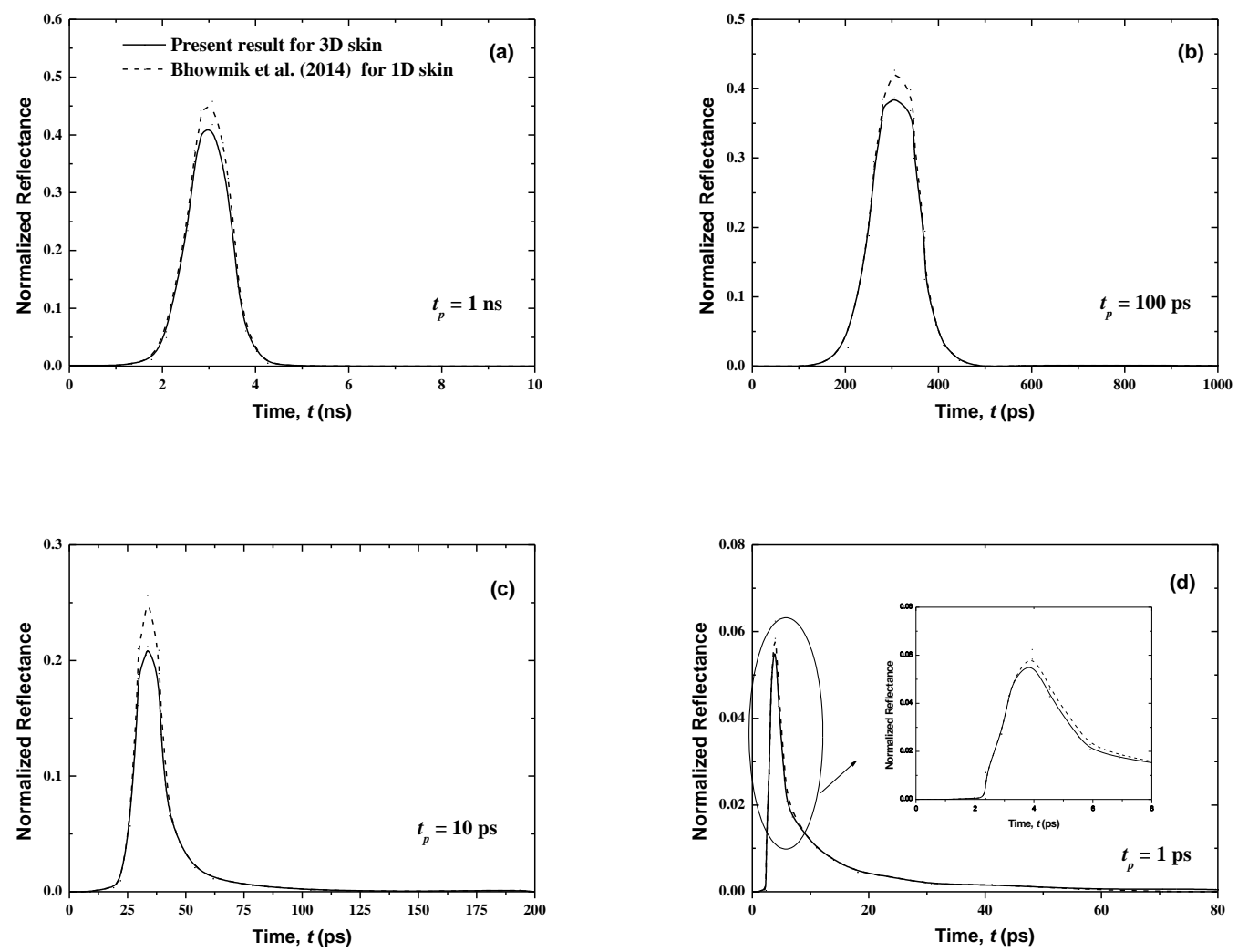

Figure 5. Temporal signals of reflectance from the normal skin subjected to the laser pulse at different pulse widths of (a) $t_{p}=1 \mathrm{~ns},(\mathbf{b}) t_{p}=100 \mathrm{ps},(\mathbf{c}) t_{p}=10 \mathrm{ps}$ and, (d) $t_{p}=1 \mathrm{ps}$.

Figures 4 and 5 show that the results of both normalized transmittance and reflectance reproduce the incident ultrafast laser, although their peak magnitudes are different. Also, the present three-dimensional signals have the same shapes as those for the one-dimensional case of [21]. For the same pulse width, the transmittance is greatly decreased (Figure 4) by comparison to the reflectance (Figure 5). In fact, for $t_{p}=1 \mathrm{~ns}$, the peak values of the normalized transmittance and reflectance are $1.8 \times 10^{-2}$ and 0.41 , respectively. It is shown that the decrease of the pulse width decreases the peak magnitude of the transmittance signals (Figure 4). This can be explained by the fact that large pulse widths require more thermal energy (heat) input to the skin, thus creating the trend obtained at higher peak amplitudes for a larger pulse width. Both peak magnitudes and temporal differences of the reflectance are small for the lower value of $t_{p}$ (Figure 5). The cascading effect becomes visible only for the lowest laser pulse width (i.e., $t_{p}=1 \mathrm{ps}$ ) (Figure 5). The consecutive augmentation in the reflectance's peak magnitude is more important for $t_{p}=1 \mathrm{ps}$.

The photons travel the skin at the speed of light within this medium, $c_{m e d}=c / n$, and thus those that are not scattered in the tissue require a time of $t=L_{\mathrm{x}} / c_{\text {med }}=37.33 \mathrm{ps}$ to reach the boundary opposite to the laser source (i.e., the boundary at $x=L_{x}$ ). Thus, the transmittance is different from zero only after this time (Figure 4); however, the reflectance, at boundary $x=0$, reveals much before this point (Figure 5). In fact, when the laser light enters the skin at the boundary $x=0$, the attenuation and diffusion of the intensity start immediately, and so an increase of the reflectance signal at this collimated boundary is obtained.

Figure 4 shows that the transmittance signals are very weak. In addition, in real medical applications, any malignancy in human skin is taken into account except for some cells of the affected tissues that are surgically removed and analyzed due to an amplified opacity of bone and skin. These bons and skin are obstacles to laser light and thus prevent transmittance signals from reaching the other side of the body. While transmittances are negligible in the case of malignancy, the reflectance 
profiles will remain present. In the next sections, only reflectance signals for malignancies in human skin are studied.

To examine the transmittance and reflectance profiles correctly, we illustrate the predictions of a laser pulse with a width of $t_{p}=1 \mathrm{ps}$ only. These predictions are easily distinguishable at smaller pulse widths, so for the evaluation objective solutions for 1 ps are discussed.

In comparison with the referenced solutions of [21], the average absolute relative error does not exceed $1.8 \%$ and $4.2 \%$ for the FT8 and FT6 schemes, respectively (Table 4). As can be seen in Table 5, an acceptable margin of error of less than $1.8 \%$ is observable for a spatial discretization of $400 \times 21 \times 21$. Thus, an excellent agreement between the present results and the ones found in the literature [21] is achieved when the FT8 scheme and a spatial discretization of $400 \times 21 \times 21$ are applied.

Table 4. Angular discretization effects on the average relative error (using $400 \times 21 \times 21$ on spatial mesh).

\begin{tabular}{cc}
\hline Angular Discretization Scheme & Average Relative Error (\%) \\
\hline FT4 & 7.28 \\
FT6 & 4.2 \\
FT8 & 1.8 \\
FT10 & 1.5 \\
\hline
\end{tabular}

Table 5. Spatial discretization effects on the average relative error (using the FT8 on angular mesh).

\begin{tabular}{cc}
\hline Spatial Discretization Scheme & Average Relative Error (\%) \\
\hline$(200 \times 11 \times 11)$ & 6.4 \\
$(200 \times 21 \times 21)$ & 5.2 \\
$(400 \times 21 \times 21)$ & 1.8 \\
$(400 \times 41 \times 41)$ & 1.3 \\
\hline
\end{tabular}

\subsection{Human Skin with Malignancies}

Skin cancer, which includes melanoma, basal cell carcinoma and squamous cell carcinoma, frequently starts as precancerous lesions. It grows through several intermediate phases and can present as new growths or precancerous lesions, whereafter it establishes itself as a tumor on the outer surface of the skin. Firstly, it is seen in the basal layer (with a thickness of around $10 \mu \mathrm{m}$ ), then it similarly develops on the other parts of neighboring cells of the epidermis layer. The neighboring medium contains the living epidermis (with a thickness of around $70 \mu \mathrm{m}$ ) and the papillary dermis (with a thickness of around $80 \mu \mathrm{m}$ ), as studied by Bhowmik et al. [21].

In the present work, consideration is given to a tool for the early detection of skin cancer, and the two intermediate phases of the development of cancer in human skin are analyzed. At first, the predictions are delivered for cases where the cancer appears only in the basal layer, and normal other layers of the human skin are conspired (Figure 6). Then, predictions are discussed for cases where the cancer develops similarly on both sides of the basal layer (i.e., the rest of the living epidermis, with a thickness of $70 \mu \mathrm{m}$ ) and the papillary dermis (with thickness: $80 \mu \mathrm{m}$ ), producing a $160-\mu \mathrm{m}$ total thickness of the affected skin tissue (Figure 7).

Figure 6 presents temporal variations in the reflectance at $x=0$ for the first case where the cancer is developed only in the basal layer (thickness: $10 \mu \mathrm{m}$ ) in consideration of the three main types of malignancies (i.e., cutaneous melanoma (CM), nodular basal cell carcinoma (NBCC) and squamous cell carcinoma (SCC)). These skin malignancies differ in their optical properties, as tabulated in Table 3. Figure 6 shows that, for normal skin, the peak magnitude of the reflectance is obtained at time $t=3.85 \mathrm{ps}$. However, the peak of magnitudes in the existence of CM, NBCC and SCC malignancies in the basal layer manifest a little ahead of the normal tissue (at $0.06,0.15$ and $0.17 \mathrm{ps}$, respectively). In addition, these peak magnitudes of reflectance are less than that of the normal skin. By comparison to the last peak magnitude cited for the normal skin, the differences are $0.00154,0.0016$ and 0.002 for $\mathrm{CM}$, NBCC and SCC, respectively (Figure 6). 

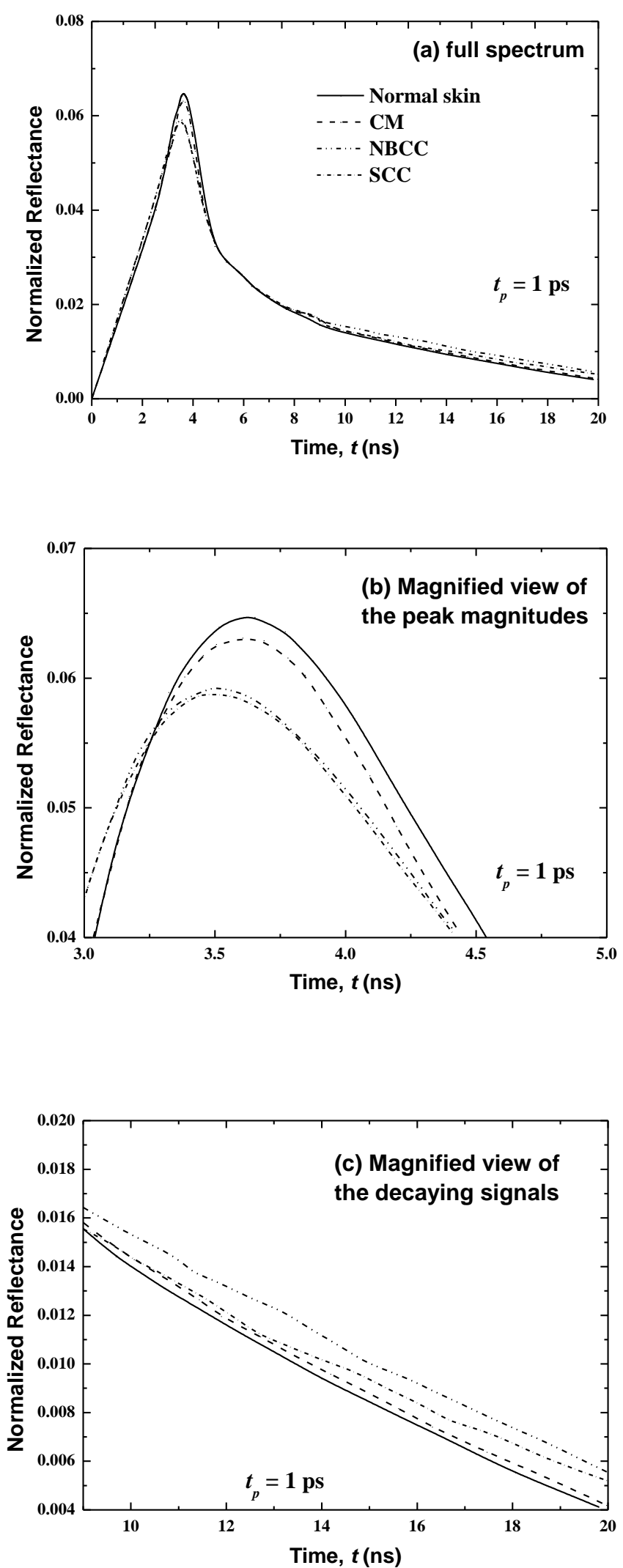

Figure 6. Temporal signals of normalized reflectance from the normal skin and skin with malignancy in the basal layer (thickness: $10 \mu \mathrm{m}$ ). 


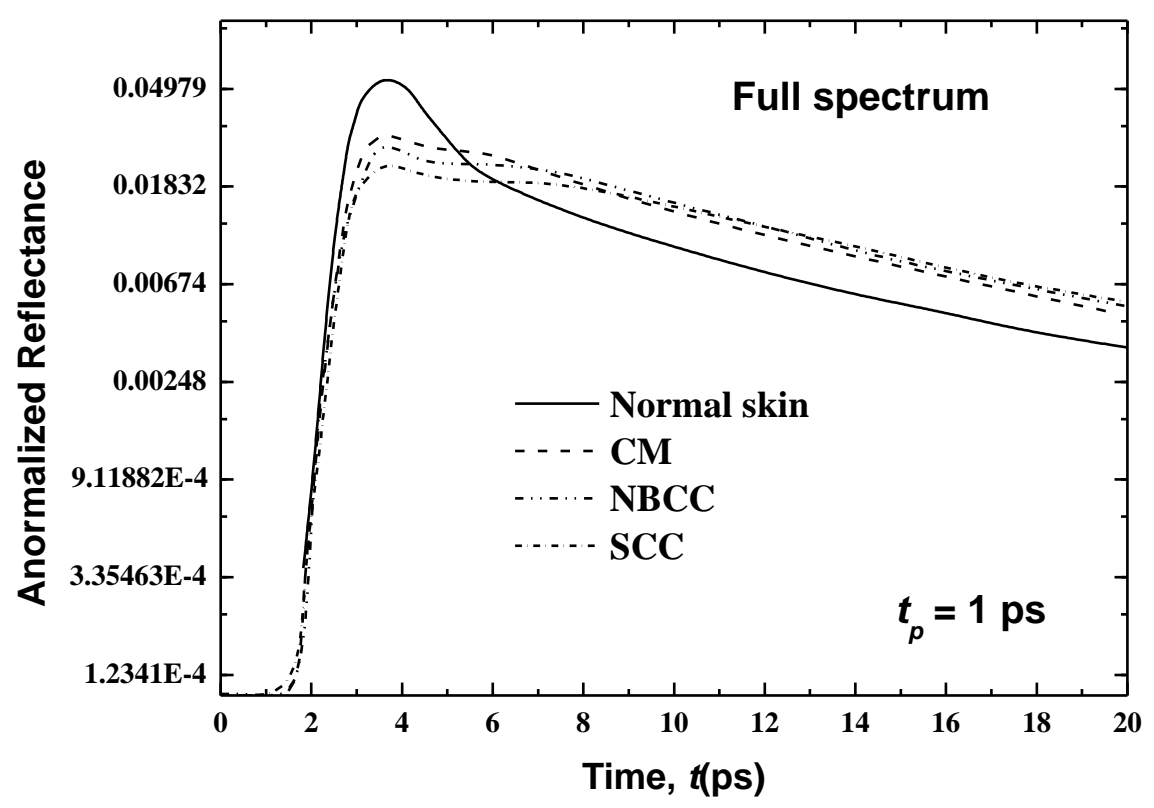

Figure 7. Temporal signals of normalized reflectance from the normal skin and skin with malignancy that has spread to the living epidermis.

In the second case, where the cancer has spread similarly on both sides of the basal layer (consisting of the living epidermis and papillary dermis) up to a total thickness of $160 \mu \mathrm{m}$, Figure 7 shows the temporal signals of the reflectance for human skin for CM, NBCC and SCC. Variances in the peak magnitudes between the normal skin and the skin with CM, NBCC and SCC malignancies are clearer than they are in the previous case (i.e., where these three types of malignancies are only located in the basal layer (Figure 6)). For the CM, NBCC and SCC malignancies, these variances are 0.026, 0.0305 and 0.0351 , respectively. Thus, the difference is approximately 16 times more than the same difference for the first case where the malignancy exists only in the basal layer (Figure 6). Similarly, this difference is also important in the decaying region (Figure 7).

It is notable that the CM, NBCC and SCC malignancies decrease the absorption coefficient and scattering coefficient, as shown in Tables 1 and 3. The emission phenomena is negligible in the case of a skin subjected to ultrafast lasers, whereas it is the scattering that gives in to the reflectance, as described above. Thus, decreasing scattering in the skin produces low magnitudes of reflectance. In addition, when this disease develops in the rest of the living epidermis as well as papillary dermis (as in the second case), the laser light scatters in additional cells $(160 \mu \mathrm{m})$ of the human tissues, so that the reflectance decreases even more.

The results show that the CM malignancy has the peak magnitude in human skin when compared against the two other malignancies, due its superior scattering coefficient (the scattering coefficients are 9.185, 8.140 and 6.680 for the skin with CM, NBCC and SCC (Table 3), respectively). It can be seen in Figures $8-11$ that for all cases of human skin with and without malignancies, the peak magnitudes occur near the cut-off time of input laser light (3 ps). 

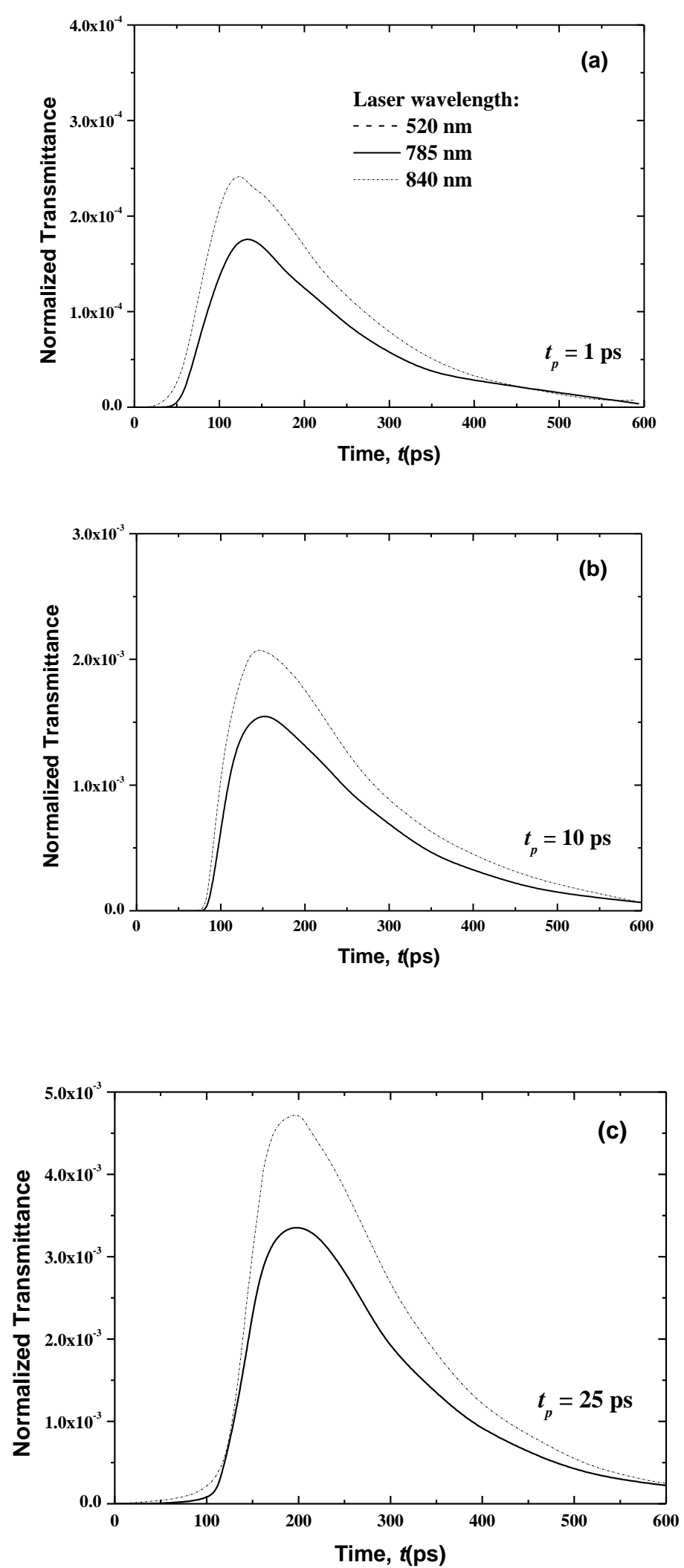

Figure 8. Temporal signals of the normalized transmittance from the normal skin at different laser pulse widths of (a) 1 ps, (b) 10 ps and (c) 25 ps. 

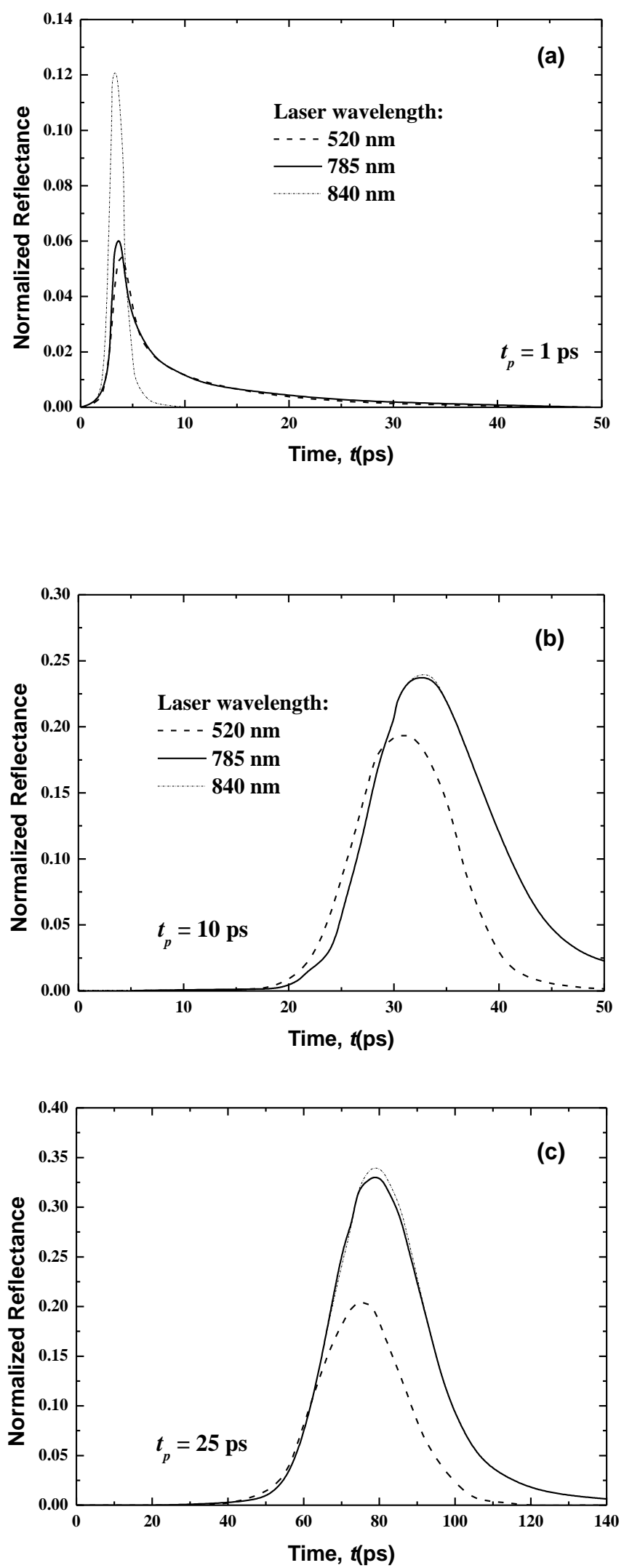

Figure 9. Temporal signals of the normalized reflectance from the normal skin at different wavelengths for laser pulse widths of (a) 1 ps, (b) 10 ps and (c) 25 ps. 


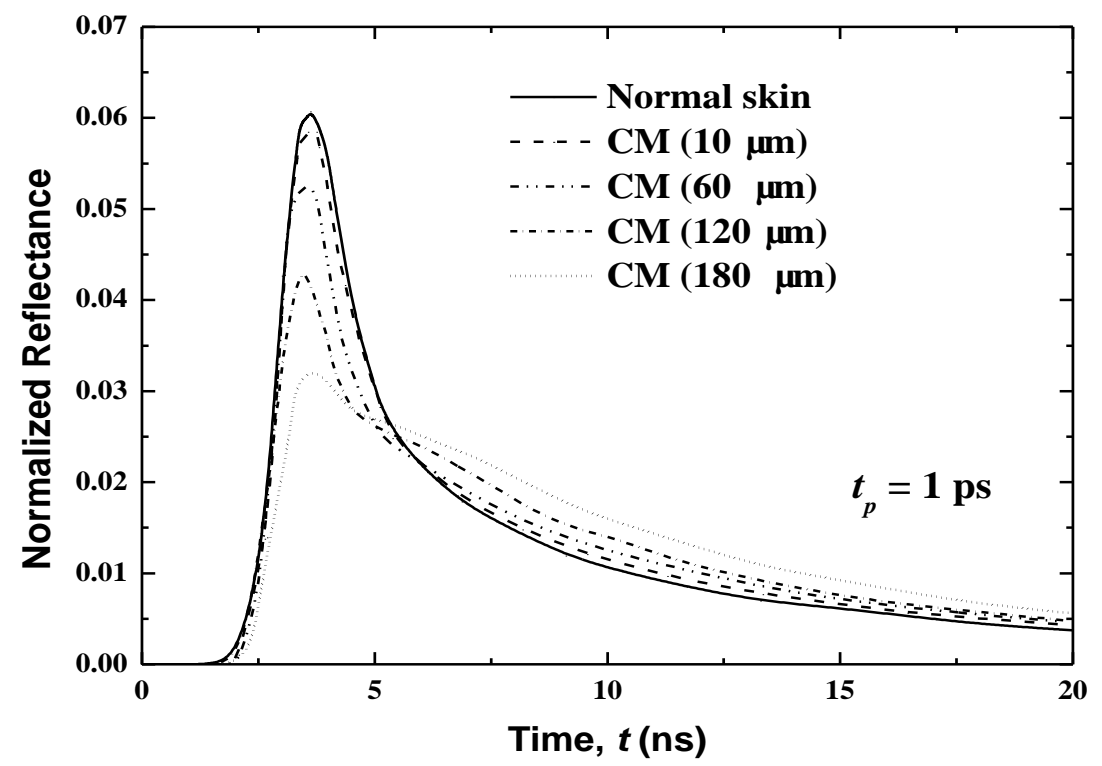

Figure 10. Effects of affected volumes on the temporal reflectance signals from the skin with CM malignancy.

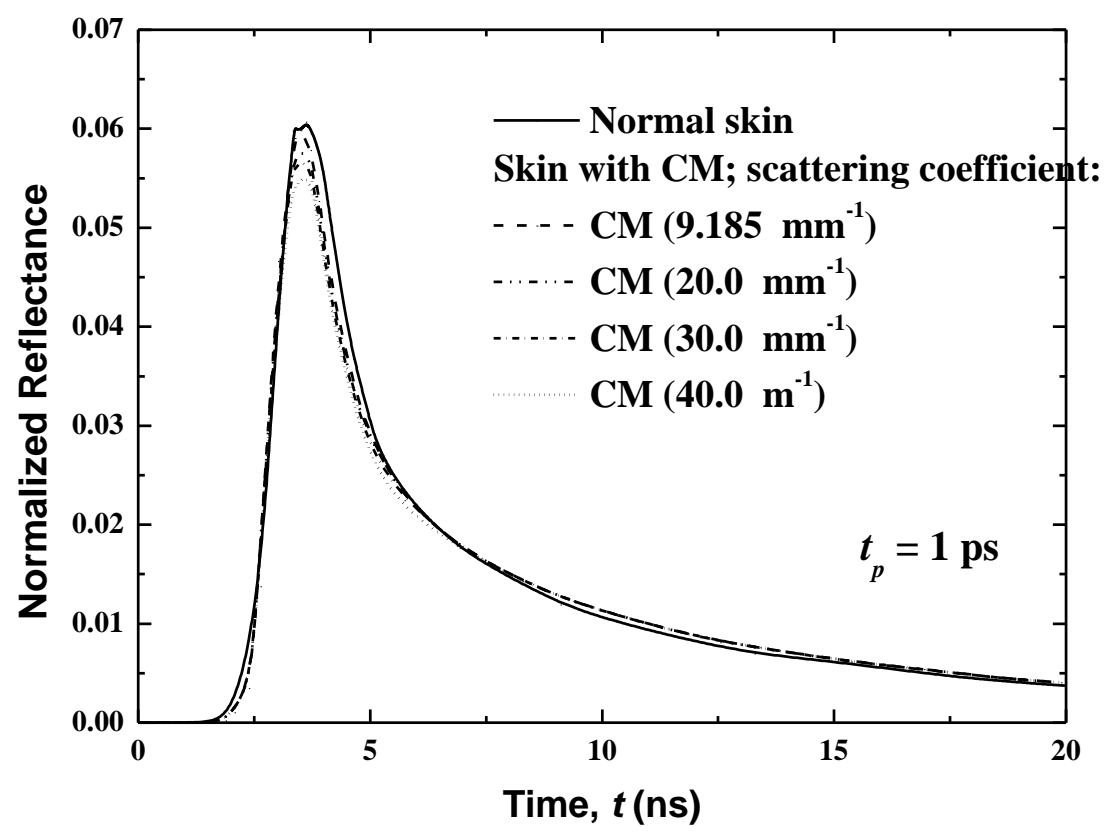

Figure 11. Effects of the scattering coefficient of the CM malignancy on the temporal reflectance signals.

\subsection{Parametric Analysis}

In this section, the effects of two main parameters are analyzed in detail: laser wavelengths, and the variations of different growth phases of cutaneous melanoma on the radiation of ultrafast laser interactions within human skin.

\subsubsection{Effects of Laser Light Wavelength}

In the present work, only three different laser wavelengths (520, 785 and $840 \mathrm{~nm}$ ) are considered. Table 1 shows the absorption coefficient, the scattering coefficient and the anisotropic factor of the human skin at $785 \mathrm{~nm}$. Table 2 summarizes these properties at 520 and $840 \mathrm{~nm}$. Additionally, three laser pulse widths (1, 10 and 25 ps) are analyzed. 
Figures 8 and 9 show the temporal transmittance and reflectance signals from normal skin subjected to a laser at these three different wavelengths and three laser pulse widths. The increase of the laser wavelength decreases the magnitudes of these two signals due to the decrease in the optical thickness of skin when the laser wavelength varies from $\lambda=840 \mathrm{~nm}$ to $\lambda=785 \mathrm{~nm}$.

In addition, laser diffuses to a greater distance at higher wavelengths at lower wavelengths, which can be shown in the difference in magnitude of the transmittance (Figure 8).

In the case of the much smaller wavelength $(\lambda=520 \mathrm{~nm})$, no transmittance signals appear at the boundary opposite to the laser source (i.e., the boundary at $x=L_{x}$ (Figure 8)). This is principally due to the attenuation of the light, which decreases with an increase of the laser wavelength, as shown in Tables 1 and 2. Furthermore, the human skin is optically thick at the smaller wavelength of $\lambda=520 \mathrm{~nm}$, so no transmittance can reach the boundary opposite the laser source. On the other hand, the reflectance signals at $x=0$, from the human skin subjected to laser light at the smaller wavelength of $\lambda=520 \mathrm{~nm}$, display an augmentation in the peak magnitude in comparison to the larger wavelengths of 785 and $840 \mathrm{~nm}$ when the laser width is 1 ps (Figure 9). For small wavelengths, most of the scattered light at the skin-surface is reflected from the stratum corneum by the laser pulse below 6 ps. Therefore, the peak magnitude of the reflectance profiles of the smaller laser wavelength $(\lambda=520 \mathrm{~nm})$ occurs before those at larger laser wavelengths $(\lambda=785$ and $840 \mathrm{~nm}$ ), as shown in Figure 8, although Figure 9 reveals that this trend in the reflectance signals is reversed for the laser pulse widths of 10 and 25 ps.

The increase of the laser pulse width above 10 ps (i.e., for 10 and 25 ps) increases the peak magnitude of the reflectance signals at the larger wavelengths ( $\lambda=785$ and $840 \mathrm{~nm})$, and these peaks become higher than the obtained value for the smaller laser wavelength $(\lambda=520 \mathrm{~nm})$. The input heat and time for which the pulse persists at any position are increased when the pulse width is above 10 ps. Therefore, the effect of the highest radiation arriving at the laser-source border from the human skin layers with a pulse width greater than $10 \mathrm{ps}$. The higher wavelength limits the outcome of the important portion of the radiation reaching the laser-source boundary from the stratum corneum. However, for the case of $\lambda=520 \mathrm{~nm}$, most of the light scatters from the stratum corneum, so the signals still seem ahead of those obtained for the two other higher wavelengths.

\subsubsection{Effect of Different Growth Phases of Cutaneous Melanoma}

Biomedical diagnosis shows that the volume of the cancerous zone increases with time. The skin cancer starts within a few cells, then grows to additional regions of the tissue, eventually reaching the phase of metastasis when it spreads to other organs of the body. Optical and thermal specifications of a skin cancer change according to its grade [31]. Further, the scattering coefficient differs over a wide range. In the present study, the effect of different growth phases of cutaneous melanoma on the laser interaction with the skin is considered. Thus, volumes of the cancerous zones are analyzed according to the varying thicknesses of the malignant layer and their scattering coefficients. CM malignancy is the most harmful malignancy, and thus attention is given in the present work to the results from $\mathrm{CM}$ malignancy specifically, with only a 785-nm laser used.

\subsubsection{Effect of the Volume of the Cancerous Region}

The effect of the cancerous region's volume is investigated through a consideration of human skin with CM that has spread to four different thicknesses: 10, 60, 120 and $160 \mu \mathrm{m}$. These thicknesses represent the spreading of $\mathrm{CM}$ in $10 \mu \mathrm{m}$ of the basal layer (case 1), $30 \mu \mathrm{m}$ of living epidermis and $30 \mu \mathrm{m}$ of papillary dermis (case 2), $30 \mu \mathrm{m}$ of living epidermis and $30 \mu \mathrm{m}$ of papillary dermis (case 3 ) and $80 \mu \mathrm{m}$ of living epidermis and $80 \mu \mathrm{m}$ of papillary dermis (case 4 ).

Figure 10 displays the temporal variations of the normalized reflectance for these four cases. In addition, the reflectance of the normal skin is also plotted. The reflectance is shown over a period of 0 to 20 ps, which is divided into two sub-periods ( $0-8$ and $8-20$ ps) to show more of the difference between signals. It is notable that the same total thickness of human skin as in the previous studies (i.e., $8.0 \mathrm{~mm}$ ) has been considered here. This shows that as the cancerous volume in the human skin 
increases, the peak magnitude of the reflectance decreases. However, an opposite trend is obtained in the decaying zone, as shown in Figure 10. The variation in the temporal profile of the normalized reflectance thus reveals the spread of cancer in more depth.

\subsubsection{Effect of the Scattering Coefficient of the Cancerous Region}

Figure 11 shows the effect of the scattering coefficient of the malignancy $\sigma_{s}$ on the temporal reflectance profiles from human skin with CM spears in the basal layer. In the present work, four different coefficients are considered: 9.185 (the same value as the previous case), 20.00, 30.00 and $40.00 \mathrm{~mm}^{-1}$. It is notable that because this layer is entirely full of this of this malignancy, the CM malignancy and basal layer have the same $\sigma_{s}$.

The modification of the scattering coefficient of the malignancy reveals a significant variation in the peak magnitude of the reflectance, as presented in Figure 11. This peak magnitude increases with an increase of $\sigma_{s}$, and while an opposite trend is obtained in the decaying region, the effect of $\sigma_{s}$ is important. The normalized reflectance is higher when the scattering coefficient of the CM is higher than that for the normal human skin, although in the case of comparable scattering coefficients, the reflectance signals are practically the same. When $\sigma_{s}$ of the CM is smaller in comparison to its value for the normal human skin, the normalized reflectance is lower. These conclusions are explained by the fact that the reflectance is principally dominated by the scattered diffuse radiation that is received at the boundary of incidence. When $\sigma_{s}$ increases, the scattering increases, and thereafter a higher reflectance is obtained. The significant augmentation of the scattering character of the damaging malignant lesion has been well-verified. This can be explained by the augmentation of the size of scatterers and/or modification of the volume density of scatterers in the affected cells $[21,27,28]$.

\section{Conclusions}

This works presents a numerical radiative heat transfer analysis of an ultrafast laser interaction with heterogeneous human tissue, with the goal of developing an optical biopsy for the early detection of skin cancer. Human brain and skin tissues subjected to ultrafast lasers have been analyzed using a formulation based on the FTn finite volume method. A combination of the curved-line advection method scheme and the FTn for spatial and angular discretization of the transient radiative transfer equation have been examined for several benchmarks.

Human skin was modeled based on the material specifics presented in other studies. Malignancies considered in the skins were cutaneous melanoma (CM), nodular basal cell carcinoma (NBCC) and squamous cell carcinoma (SCC). In the initial phase of the skin cancer, the above malignancies were situated in the basal layer, after which they were developed in the full living epidermis. Predictions showed that transmittance decreases with an increase of the extinction coefficient, and that it becomes very weak with a higher extinction coefficient. Reflectance signals are important due to strongly scattered human skin. Therefore, the effects of short-pulse laser widths were investigated.

Malignancies in the basal layer and living epidermis were considered. Further, the effects of laser light wavelength, the volume of the cancerous region and the scattering coefficient on their temporal signals of reflectance and transmittance were studied and compared against those for normal human skin (i.e., without malignancies). The results demonstrated that in the maximum zone a high reflectance was obtained for human skin with CM, while a low reflectance was observed in the case of skin with SCC. When living epidermis was complete infected with any of these malignancies, the reflectance was more than its values predicted in the case of infection of the basal layer on its own. Further, the effects of laser light wavelength, the volume of the cancerous region and its scattering coefficient on reflectance and transmittance were studied.

Such predictions reported here for nonhomogeneous human skin tissues with a small cancerous zone subjected to an ultrafast laser may be explored for the early detection of small cancerous tumors. In fact, the obtained results from the radiative signals of skins with cutaneous melanoma, nodular basal cell carcinoma or squamous cell carcinoma are clearly different from normal skin, allowing an early 
detection of skin cancer. The reflectance of human skin with these types of cancer also changes from stage to stage as a result of the carcinoma thickness. Lower and slightly different profile peaks were obtained with increases of this thickness. Also, for cancerous skin, the results demonstrated a small advancement of the peaks and a presence of a second local profile maximum in more advanced stages that could allow a good and early identification of skin cancer. Thus, the obtained modifications of the optical properties and conditions represent a potential for the early detection of skin cancer. Further complete theoretical, numerical and experimental research will be surveyed soon. Moreover, in future works, the effects of optical characteristics of the granules will be introduced in the proposed mathematical formulation for improving the numerical accuracy of the present tool.

Author Contributions: Formal analysis, K.G. and M.O.; Funding acquisition, S.S.A.; Methodology, K.G., M.O. and S.S.A.; Visualization, K.G., M.O. and S.S.A.; Writing-original draft, K.G. and M.O.; Writing-review \& editing, K.G. All authors have read and agreed to the published version of the manuscript

Funding: This work was supported by the Deanship of Scientific Research (DSR), King Abdulaziz University, Jeddah, under grant No (DF-191-980-1441). The authors therefore gratefully acknowledge DSR technical and financial support.

Acknowledgments: The authors gratefully acknowledge DSR, King Abdulaziz University, technical and financial support.

Conflicts of Interest: The authors declare no conflict of interest.

\section{Nomenclature}

c speed of light

CM cutaneous melanoma

I intensity

$I_{b} \quad$ black body intensity

$I_{c} \quad$ collimated intensity

$I_{d} \quad$ diffuse component of the intensity

$g \quad$ anisotropic factor

$L \quad$ total number of discrete solid angles

NBCC Nodular Basal Cell Carcinoma

SCC Squamous Cell Carcinoma

$S_{c} \quad$ source term due to collimated radiation

$S_{d} \quad$ source term due to diffuse radiation

$t$ time

$T$ temperature

Greek symbols

$\beta \quad$ extinction coefficient

$\Delta \Omega \quad$ control solid angle

$\varepsilon_{w} \quad$ emissivity

$\Phi \quad$ scattering phase function

$\sigma_{a} \quad$ absorption coefficient

$\sigma_{s} \quad$ scattering coefficient

$\tau \quad$ nondimensional optical thickness

$\omega \quad$ scattering albedo

$\Omega \quad$ unit vector in direction of intensity

Superscripts

$l, l^{\prime} \quad$ discrete angular directions

\section{References}

1. Akamatsu, M.; Guo, Z. Ultrafast Laser Pulse Train Radiation Transfer in a Scattering-Absorbing 3D Medium with an Inhomogeneity. Heat Transf. Res. 2015, 46, 861-879. [CrossRef]

2. Guo, Z.; Kumar, S.; San, K.-C. Multidimensional Monte Carlo Simulation of Short-Pulse Laser Transport in Scattering Media. J. Thermophys. Heat Transf. 2000, 14, 504-511. [CrossRef] 
3. Jang, C.; Jung, H.; Lee, J.; Song, T.-H. Radiative heat transfer analysis in pure scattering layers to be used in vacuum insulation panels. Appl. Energy 2013, 112, 703-709. [CrossRef]

4. Wang, C.-H.; Zhang, Y.; Yi, H.-L.; Tan, H.-P. Transient radiative transfer in two-dimensional graded index medium by Monte Carlo method combined with the time shift and superposition principle. Num. Heat Transfer Part A 2016, 69, 574-588. [CrossRef]

5. Tan, Z.-M.; Hsu, P.-F. An Integral Formulation of Transient Radiative Transfer. J. Heat Transf. 2000, 123, 466-475. [CrossRef]

6. Hunter, B.; Guo, Z. Improved Treatment of Anisotropic Scattering for Ultrafast Radiative Transfer Analysis. J. Heat Transf. 2015, 137, 091004. [CrossRef]

7. Hunter, B.; Guo, Z. Numerical smearing, ray effect, and angular false scattering in radiation transfer computation. Int. J. Heat Mass Transf. 2015, 81, 63-74. [CrossRef]

8. Coelho, P.J. Bounded Skew High-Order Resolution Schemes for the Discrete Ordinates Method. J. Comput. Phys. 2002, 175, 412-437. [CrossRef]

9. Guedri, K.; Bouzid, A.; Nasr, A.; Al-Ghamdi, A.S. Three-dimensional FTn Finite Volume Analysis of Short Pulse Laser Propagation through Biomedical Tissue Phantoms. Int. J. Applied Eng. Res. 2018, 13, 4764-4775.

10. Ettaleb, A.; Ammar, A.M.; Farhat, H.; Guedri, K.; Omri, A.; Borjini, M.N.; Goodarzi, M.; Sarafraz, M. Radiation Heat Transfer in a Complex Geometry Containing Anisotropically-Scattering Mie Particles. Energies 2019, 12, 3986. [CrossRef]

11. Guedri, K.; Al-Ghamdi, A.S. Radiative Heat Transfer in Complex Enclosures Using NVD Differencing Schemes of the FTn Finite Volume Method. Heat Transf. Res. 2017, 48, 1379-1398. [CrossRef]

12. Guedri, K.; Al-Ghamdi, A.S. Improved Finite Volume Method for Three-Dimensional Radiative Heat Transfer in Complex Enclosures Containing Homogenous and Inhomogeneous Participating Media. Heat Transf. Eng. 2017, 39, 1364-1376. [CrossRef]

13. Guedri, K.; Al-Ghamdi, A.S.; Oreijah, M. Application of high-resolution NVD differencing schemes to the FTn finite volume method for radiative heat transfer. Heat Transfer-Asian Res. 2017, 47, 366-388. [CrossRef]

14. Guedri, K.; Bouzid, A.; Nasr, A.; Al-Ghamdi, A.S. Three-dimensional FTn finite volume solution of short-pulse laser propagation through heterogeneous medium. Therm. Sci. 2018, 24, 186. [CrossRef]

15. Park, H.; Chacón, L.; Matsekh, A.; Chen, G. A multigroup moment-accelerated deterministic particle solver for 1-D time-dependent thermal radiative transfer problems. J. Comput. Phys. 2019, 388, 416-438. [CrossRef]

16. Baek, S.W.; Byun, D.Y.; Kang, S.J. The combined Monte-Carlo and finite-volume method for radiation in a two-dimensional irregular geometry. Int. J. Heat Mass Transf. 2000, 43, 2337-2344. [CrossRef]

17. Chai, J.C.; Moder, J.P. Radiation Heat Transfer Calculation Using an Angular-Multiblock Procedure. Numer. Heat Transf. Part B Fundam. 2000, 38, 1-13. [CrossRef]

18. Kim, S.H.; Huh, K.Y. A new angular discretization scheme of the finite volume method for 3-D radiative heat transfer in absorbing, emitting and anisotropically scattering media. Int. J. Heat Mass Transf. 2000, 43, 1233-1242. [CrossRef]

19. Guo, Z.; Kumar, S. Discrete-ordinates solution of short-pulsed laser transport in two-dimensional turbid media. Appl. Opt. 2001, 40, 3156-3163. [CrossRef]

20. Hiroyuki, F.; Moegi, U.; Kazumichi, K.; Masao, M. Characteristic Length and Time Scales of the Highly Forward Scattering of Photons in Random Media. Appl. Sci. 2020, 10, 93.

21. Bhowmik, A.; Repaka, R.; Mishra, S.C.; Mitra, K. Analysis of radiative signals from normal and malignant human skins subjected to a short-pulse laser. Int. J. Heat Mass Transf. 2014, 68, 278-294. [CrossRef]

22. Guo, Z.; Kim, K. Ultrafast-laser-radiation transfer in heterogeneous tissues with the discrete-ordinates method. Appl. Opt. 2003, 42, 2897-2905. [CrossRef] [PubMed]

23. Guedri, K.; Al-Ghamdi, A.S.; Bouzid, A.; Abbassi, M.A.; Ghulman, H.A. Evaluation of the FT n Finite Volume Method for Transient Radiative Transfer in Anisotropically Scattering Medium. Numer. Heat Transf. Part A Appl. 2015, 68, 1137-1154. [CrossRef]

24. Salomatina, E.; Jiang, B.; Novak, J.; Yaroslavsky, A.N. Optical properties of normal and cancerous human skin in the visible and near-infrared spectral range. J. Biomed. Opt. 2006, 11, 064026. [CrossRef]

25. Garcia-Uribe, A.; Smith, E.B.; Zou, J.; Duvic, M.; Prieto, V.; Wang, L.V. In-vivo characterization of optical properties of pigmented skin lesions including melanoma using oblique incidence diffuse reflectance spectrometry. J. Biomed. Opt. 2011, 16, 020501. [CrossRef]

26. Anderson, R.R.; Parrish, J.A. The Optics of Human Skin. J. Investig. Dermatol. 1981, 77, 13-19. [CrossRef] 
27. Bashkatov, A.N.; Genina, E.A.; Kochubey, V.I.; Tuchin, V.V. Optical properties of human skin, subcutaneous and mucous tissues in the wavelength range from 400 to $2000 \mathrm{~nm}$. J. Phys. D Appl. Phys. 2005, 38, 2543-2555. [CrossRef]

28. Bashkatov, A.N.; Genina, E.A.; Tuchin, V.V. Optical Properties of Skin, Subcutaneous, and Muscle Tissues: A Review. J. Innov. Opt. Health Sci. 2011, 4, 9-38. [CrossRef]

29. Simpson, R.C.; Kohl, M.; Essenpris, E.; Cope, M. Near-infrared optical properties on ex vivo human skin and subcutaneous tissues measured using Monte Carlo inversion technique. Phys. Med. Biol. 1998, 43, 2465-2478. [CrossRef]

30. Wang, S.; Zhong, S.-L.; Lui, H.; He, Q.; Zeng, H. Monte Carlo simulation of near infrared autofluorescence measurements of in vivo skin. J. Photochem. Photobiol. B Biol. 2011, 105, 183-189. [CrossRef]

31. Cheong, W.F.; Prahl, S.A.; Welch, A.J. A review on optical properties of biological tissues. IEEE J. Quantum Electron. 1990, 26, 2166-2184. [CrossRef]

(C) 2020 by the authors. Licensee MDPI, Basel, Switzerland. This article is an open access article distributed under the terms and conditions of the Creative Commons Attribution (CC BY) license (http://creativecommons.org/licenses/by/4.0/). 